\section{Servants to two masters}

If genes had ambition, then they would probably aspire to being a 'master' gene - one whose presence is necessary and sufficient to initiate the chain of events that specify the fate of a tissue or organ. Alas, for every tissue that needs to be specified there can be, by definition, only one master gene. For those studying the determination of the eye field in Drosophila, adhering to this definition has not been easy. Over the past few years, seven genes (eyeless, twin of eyeless, eyes absent, sine oculis, dachschund, eye gone and optix) have been assigned the master role in initiating eye cell fate. In each case, the claim for master status has been justified according to the criteria that were used to define it: no eye forms when any one of these genes is mutated and, conversely, an ectopic eye can form when any one of them (except sine oculis) is overexpressed in non-eye tissue. The eye community therefore faced a logical impossibility — one master gene and seven, seemingly valid, candidates.

So who is the master? To try to answer this, Kumar and Moses have done what is required when the experimental data contradicts the assumption - they questioned the assumptions. The assumptions in question are the criteria used to define a master gene. Such a gene, the authors posit, should be expressed in one tissue during development, specifically in the tissue that it presumably specifies, and its absence would cause the homeotic transformation of that tissue into another. Mutations in any of the seven genes cause the degeneration of the eye disc (the larval structure that gives rise to the adult eye), but never a homeotic change. Furthermore, these genes are never co-expressed in the embryo - which is what you'd expect if, as genetic evidence indicates, they function as a molecular complex. As none of the seven genes listed above satisfies these criteria, what does? The authors, who report their findings in Cell, show that two molecules that are well known to control many aspects of eye development, the transmembrane receptor Notch and the Epidermal growth factor receptor (Egfr), can act antagonistically as homeotic determinants of eye specification. Hyperactivation of the Egfr or downregulation of Notch signalling in the eye disc is sufficient to formed antenna. The two receptors are known to antagonize each other at many stages of fly development, and their relationship is recapitulated here, with the Egfr promoting antennal fate, and Notch promoting eye fate. convert the eye field into a perfectly
Having satisfied the criterion of homeosis, do the Egfr and Notch satisfy the second condition, that of being expressed at the time when eye fate is specified? To establish this it was necessary to determine when the eye field is specified. Contrary to previous reports, the crucial period for establishing eye fate occurs not in embryogenesis but later, during the second larval moult. The expression of Notch is upregulated in the eye disc at the second-thirdinstar transition, lending further support to the theory. Furthermore, it is at this stage that Notch induces the concerted expression of all seven genes in the eye.

The story comes together neatly in a simple model: signalling by the Egfr and Notch at the second-thirdmoult transition concentrates the expression of the eye-specifying complex of genes, thus determining the eye fate.

This could be the first time that a homeotic function has been assigned to a receptor tyrosine kinase. This study hasn't revealed the long-sought-after master gene for eye fate but, by looking upstream of Notch and of the Egfr, the servants of eye research might find they are only one step away.

Tanita Casci

(2) References and links ORIGINAL RESEARCH PAPER Kumar, J. P. \& Moses, K. EGF receptor and Notch signaling act upstream of Eyeless/Pax6 to control eye specification. Cell 104, 687-697 (2001) FURTHER READING Gehring, W. J. Master Control Genes in Development and Evolution: The Homebox Story (Yale University Press, New Haven, Connecticut, 1998) WEB SITE Kevin Moses' lab
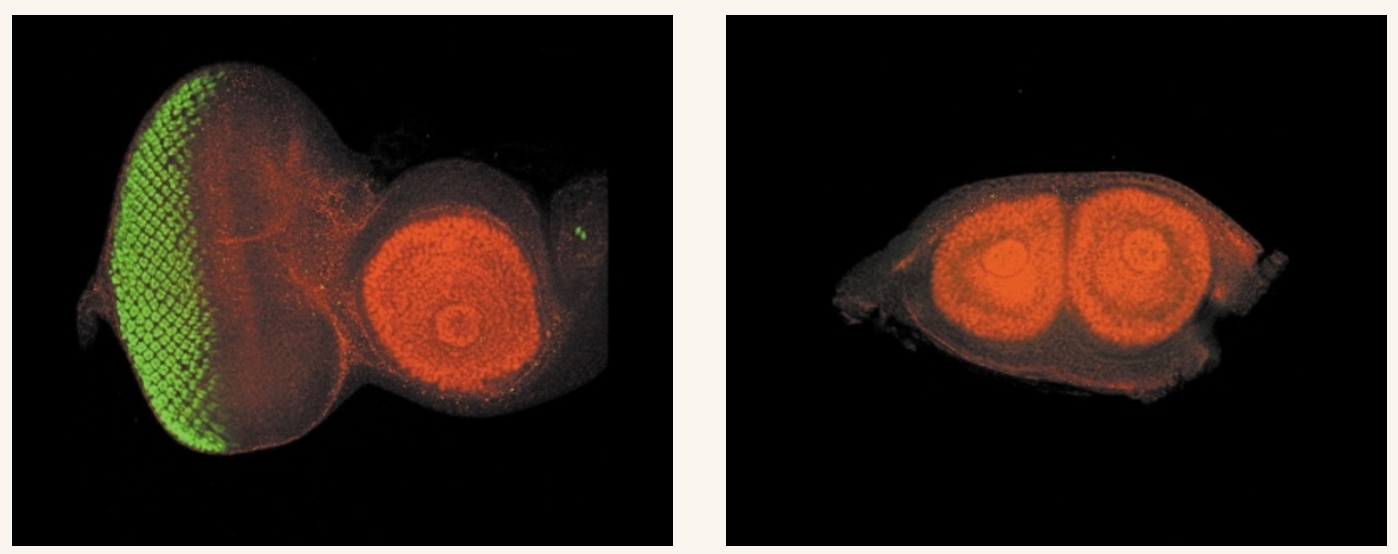

An eye and an antennal imaginal disc (left). The photoreceptors are shown in green. Homeotic transformation of the eye field into an antennal field after loss of Notch activity at the second instar stage (right). Reprinted from Cell 104, 687-697 @ (2001), with permission from Elsevier Science.

\section{WEB WATCH}

E-access to science If there is one debate that has dominated labs, libraries and scientific publishers lately, it is that surrounding the call for unrestricted access to primary scientific research publications, which, according to its proponents, should be housed in a centralized online database. The initiative, known as the 'The Public Library of Science' (PLS) has attracted a wide range of opinions and has stimulated heated discussion over ownership of research results, and on the practica and ethical consequences of storing scientific data in a single repository.

More generally, this initiative fits into the larger question of the impact of electronic publishing on science. Does the future of science publishing belong online? If so, how will the transition from print to Web publishing occur? What kind of socioeconomic and technical questions should be addressed? Who will ensure quality controls on scientific information?

Nature has now brought this debate online. From the 6 April 2001, scientists can become better acquainted with the issues of this debate through articles written by publishers, librarians and technology developers. Access is free, and the Web debate can be reached directly or from the Nature home page. The featured articles cover a selection of viewpoints, from those who believe that scientific information belongs to those who generate it, to those who fear that the PLS project would harm science.

Visitors to the Web forum can also express their own opinions and reactions to this debate by sending their views to be posted on this site. As the curators point out, this debate will not resolve all the controversies, but it might help publishers and scientists to identify how to handle and disseminate scientific information in the most effective manner.

Tanita Casci 